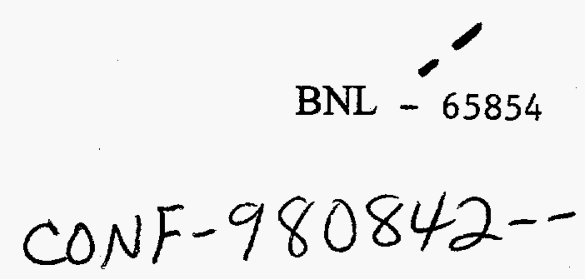

Power Spectrum Calculation for the Cornell Wiggler

A SASE Experiment at BNL

Li Hua Yu

National Synchrotron Light Source, Brookhaven National Laboratory

P.O. Box 5000, Upton, NY, USA 11973-5000

August 1998

National Synchrotron Light Source

RECEIVED

NOV 201998

Brookhaven National Laboratory

Operated by

Brookhaven Science Associates

OSTI

Upton, NY 11973

Under Contract with the United States Department of Energy

Contract Number DE-AC02-98CH10886

OISTRIBUTION OF THIS DOCUMENT IS UMUMIED

MASTER 


\section{DISCLAIMER}

This report was prepared as an account of work sponsored by an agency of the United States Government. Neither the United States Government nor any agency thereof, nor any of their employees, nor any of their contractors, subcontractors or their employees, makes any warranty, express or implied, or assumes any legal liability or responsibility for the accuracy, completeness, or any third party's use or the results of such use of any information, apparatus, product, or process disclosed, or represents that its use would not infringe privately owned rights. Reference herein to any specific commercial product, process, or service by trade name, trademark, manufacturer, or otherwise, does not necessarily constitute or imply its endorsement, recommendation, or favoring by the United States Government or any agency thereof or its contractors or subcontractors. The views and opinions of authors expressed herein do not necessarily state or reflect those of the United States Government or any agency thereof. 


\section{DISCLAIMER}

Portions of this document may be illegible in electronic image products. Images are produced from the best available original document. 


\title{
Power Spectrum Calculation for the Cornell Wiggler A SASE Experiment at
} BNL

\author{
Li Hua Yu \\ National Synchrotron Light Source, Brookhaven National Laboratory, Upton, N.Y.11973
}

\begin{abstract}
Recently we showed [1] that the widely used simulation code TDA3D, even though a single frequency code, can be used to determine the power spectrum in the SASE process with excellent approximation in the exponential growth regime. In this paper, we apply this method to the BNL Cornell Wiggler A SASE experiment as an example.

When the gain is not very high, there are many modes in the radiation, which seems to make the analytical calculation very difficult. However, we show that the increment of the radiation due to SASE over the spontaneous radiation can be expanded in terms of guided modes with rapid convergence. Thus when the spontaneous radiation is substracted from the SASE power during the calculation, there is a good agreement between the analytical theory and the numerical simulation .
\end{abstract}

\section{Introduction}

Recently, we showed [1] that the TDA3D code, which has been modified to include harmonic generation calculation, can be used to calculate the power spectrum. One reason that made this possible is that in the linear regime there is a very simple scaling relation between the number of simulation particles and the output power: the output power is inversely proportional to the number of simulation particles. Hence, the number of simulation particles can be made much smaller than the actual number of electrons in the beam, making the simulation practical.

This method uses an entirely different approach to reduce the number of simulation particles as compared with the simulation codes such as GINGER. We shall briefly compare these 
two approaches. The codes such as GINGER use an artificial initial distribution to simulate the SASE start-up process. To suppress the increased shot noise due to the limited number of simulation particles, the codes are based on a distribution with equally spaced particles. To introduce a controlled noise, they generate a random deviation from the equally spaced distribution with a controlled rms value of the displacements. The rms displacement is chosen to reproduce the same mean and variance of the bunching parameter. While the mean and variance of the bunching parameter simulate the initial status of the system, it is not evident that the higher moments of this quantity would not affect the high gain process, it is also not evident that the mean and variance of the relevant quantity would remain to be the same as the realistic distribution during the high gain process, even though the simulations did show an agreement with the linear high gain theory.

As compared with these codes, our method uses a realistic distribution instead of an artificial evenly spaced distribution. We do not attempt to suppress the noise due to the limited number of simulation particles. Rather, use the scaling relation to go from the simulation case with increased start-up noise (due to the reduced number of simulation particles) to the realistic case. In this manner, we obtain the correct radiation power in the linear regime.

Another reason that we can use a single frequency code such as TDA3D to calculate the intrinsically broad band SASE process is the following. In the original TDA code, all the simulation particles are limited to within one optical wavelength, or, one cell. During a later modification of the code for harmonic generation calculation, we extended the code such that the simulation particles could be in an arbitrary number of wavelengths. It is easy to see that if the number of cells is $n_{l}$, then the code is describing a fictitious electron beam distribution with longitudinal periodic structure of $n_{l}$ optical wavelengths. That is, we artificially set a periodic boundary condition on the electron beam with period equal to $n_{l}$ optical wavelengths. In this case, the radiation spectrum has a line structure with frequency spacing $\omega_{s} / n_{l}$, where 
$\omega_{s}$ is the optical frequency.

We denote the slippage distance by $l_{s}=N_{w} \lambda_{s}$, and the distribution period by $l=n_{l} \lambda_{s}$, then when $l \gg l_{s}$, the line spacing $\omega_{s} / n_{l}$ is much narrower than the spontaneous radiation width $\omega_{s} / N_{w}$ and the dense line structure gives a profile of the spontaneous spectrum. When we choose the period to be equal to the slippage distance $l=l_{s}$, i.e., when $n_{l}=N_{w}$, the line spacing is equal to the radiation spectrum width, and hence there is only one line. The slippage $N_{w} \lambda_{s}$ is equal to the spacing between the periodic boundaries of the electron beam. Hence there is no interaction between any two of the idealized periods of the electron distribution. The calculated output energy within one idealized period of the electron distribution is the same as it would be from a non-periodic structure in the electron beam, i.e., the same as for the realistic case for SASE process. The output power is shown to be $\frac{1}{N_{w}}\left(\frac{\partial P_{\text {spon. }}}{\frac{\partial \omega}{\omega}}\right)$.

Now from the 1D analytic theory of SASE, we know that the full bandwidth of the SASE spectrum is [1] $\left(1 / N_{w}\right) \cdot \sqrt{L_{w} 3 / 4 \pi L_{G}} \cong\left(1 / N_{w}\right) \cdot \sqrt{L_{w} / 4 L_{G}}$. This width is narrower than $2 / N_{w}$ as long as $L_{w}<16 L_{G}$. So when the wiggler length is much shorter than 16 power gain lengths, if we choose $n_{l}$ to be equal to the number of periods $N_{w}$, to good approximation there is only one line within the bandwidth centered around the resonant frequency. Therefore, when the wiggler length is much shorter than 16 power gain lengths TDA3D serves as a good approximation to the output power even though it handles only one single spectral line. In this paper we assume the electron bunch is much longer than the slippage and the bunch shape is sufficiently smooth.

In section 2, we shall apply the new method to simulate the spontaneous radiation power spectrum in the BNL Cornell Wiggler A experiment as an example and a check of the calculation. In section 3, we apply the method to the SASE calculation of the same experiment, and describe the analytical calculation.

2. The calculation of the spontaneous radiation spectrum 
We consider the parameters for the BNL Cornell Wiggler A SASE experiment: the radiation resonant wavelength is $\lambda_{s}=5 \mu \mathrm{m}$, the wiggler period is $\lambda_{w}=3.3 \mathrm{~cm}$, the wiggler length is $L_{w}=1.98 \mathrm{~m}$, the number of period is $N_{w}=60$, with wiggler parameter $K_{\max }=1.44$, and the e-beam energy is $\gamma=82$. We take a small current $I_{0}=10$ ampere to calculate spontaneous radiation. Our analytical calculation based on the well-known spontaneous radiation theory shows that the power spectrum, integrated over the full solid angle of the radiation, is as shown by the solid curve in Figure 1. Because when the radiation angle is deviating from the forward direction, the wavelength is always shifted to longer, even though for a very small solid angle the radiation spectrum is a simple sinc function of width $1 / N_{w}$, the integrated spectrum over all angle is more like a step function with rising width $1 / N_{w}$ near the resonant wavelength $\lambda_{s}$. We can show that the peak of the power spectrum, integrated over the full solid angle, is given by:

$$
\left(\frac{\partial P_{\text {spon. }}}{\frac{\partial \omega}{\omega}}\right)_{\text {peak }} \cong \pi \theta_{w}^{2} B_{0}
$$

where $\theta_{w} \equiv \sqrt{2 \lambda_{s} / L_{w}}$ is defined as the opening angle of the spontaneous radiation within a sufficiently narrow bandwidth, and $B_{0}$ is the brightness in the forward direction and at the resonant frequency, given by:

$$
B_{0}=\left(\frac{\partial^{2} P_{s p m t}}{\frac{\partial \omega}{\omega} \partial \Omega}\right)_{\theta=0 \omega=\omega_{s}}=\frac{e Z_{0} I_{0}}{4 \pi} N_{w}^{2} \gamma^{2} \frac{K_{\max }^{2}}{\left(1+\frac{K_{\max }^{2}}{2}\right)^{2}}[J J]^{2} \omega_{s}
$$

with $Z_{0}=377 \Omega$ the vacuum impedance, and $[J J]$ the Bessel factor. We can also show that at the resonant wavelength $\lambda_{s}$, the power spectrum is half of the peak height, and the peak is positioned at a longer wavelength, away from $\lambda_{s}$ by a space of order but less than $\lambda_{s} / N_{w}$. For our example, these formulas give $\theta_{w}=2.2 \mathrm{mrad}$ and $\frac{1}{N_{w}}\left(\frac{\partial P_{\text {spon. }}}{\frac{\partial \omega}{\omega}}\right)_{\text {peak }}=0.043$ watt.

As explained before, to calculate the power spectrum, we choose the number of wavelength cells in the TDA3D calculation to be $N_{w}=60$. The number of electrons in one wavelength is 
then $N=I_{0} \lambda_{s} / e c=1.1 \times 10^{6}$. Because the algorithm used by TDA3D [5], we must always specify an input power to normalize the calculation, we choose the input power to be $10^{-10}$ watt, which is small enough that the output is entirely determined by the shot noise of the simulation particle, and not affected by this number. When the number of simulation particles is $N^{\prime}=1200$, for the given parameters, we found that at $\lambda=5.05 \mu m$, the radiation power reaches the peak value of $P^{\prime}=40$ watt, after averaging over many runs. Thus, using the scaling relation, we find that the real radiation power spectrum is

$$
\frac{1}{N_{w}}\left(\frac{\partial P_{\text {spon. }}}{\frac{\partial \omega}{\omega}}\right)_{\text {peak }}=\frac{N^{\prime}}{N}<P^{\prime}>=\frac{1200}{1.1 \times 10^{6}} \times 40=0.04 \text { watt }
$$

To test the convergence of the simulation, the figure 1 gives the results for several sets of simulation of the power spectrum as a function of the wavelength $\lambda$ and compare them with the analytical theory. The figure also indicates the number of runs of TDA3D for each of the average points. The figure shows that when the number of radial mesh points NPTR $=360$ and average over 20 runs, the simulation is converging to the analytical theory.

\section{The simulation of SASE}

To simulate SASE, we assume an idealized distribution used in [2]. The transverse distribution is a step function profile with a constant current density within a radius of $\sqrt{6} \sigma_{x}$, where $\sigma_{x}=170 \mu \mathrm{m}$ is the rms radius, the current density is zero outside this radius. We assume all the electron momentum is parallel to the wiggler axis. We assume both the horizontal and vertical focusing is zero. Use the method described in section 2, we plot in figure 2 the power spectrum at the resonant frequency $\omega_{s}$ as a function of current, varied from zero to 110 ampere, every point is an average over 10 run with different initial random number seeds. In this calculation we used 5 azimuthal modes with $\mathrm{m}=0, \pm 1, \pm 2$.

For this idealized model, the SASE power in the guided modes is explicitly solved $[2,3]$. The power spectrum in a mode $n=\{j, m\}$ ( $j$ is the radial node number, $m$ is the axial node 
number) is:

$$
\left(\frac{d P_{n n}}{d \omega}\right)_{S A S E}=\frac{1}{9} e^{\frac{L_{w}}{L_{G_{n}}}} C_{n}(\tilde{a})\left[\frac{2 L_{G_{n}}}{L_{w}}\left(\frac{d P}{d \omega}\right)_{S p m .}^{L_{w}}\right]
$$

where $L_{G_{n}}$ is the power gain length, and $\widetilde{a}$ is the scaled beam size defined by $\widetilde{a}^{2}=8 k_{s} k_{w} \rho \sigma_{x}$, with $k_{s}, k_{w}$ the wavenumber for the radiation and the wiggler respectively. $\rho$ is the Pierce parameter [4] given by

$$
(2 \rho)^{3}=\frac{n_{0} Z_{0} e^{2} K_{r m s}^{2}[J J]^{2}}{2 m \gamma^{3} k_{w}^{2} c},
$$

where $n_{0}$ is the peak current density, in our case it is just the current density within the edge radius $\sqrt{6} \sigma_{x}$, since it is a constant. $K_{r m s}$ is the rms wiggler parameter. The gain length is given by $L_{G_{n}}=\lambda_{w} / 8 \pi \rho \operatorname{Im}\left(\lambda_{n}(\tilde{a})\right)$. Thus the power spectrum Eq.(4) is completely determined by the scaled beam size $\tilde{a}$ through two functions: the coupling coefficient $C_{n}$ and scaled growth rate $\lambda_{n}$. The physical meaning of Eq.(4) is clear now: the SASE input noise is the spontaneous radiation power spectrum within two power gain lengths $\left[\frac{2 L_{G_{n}}}{L_{w}}\left(\frac{d P}{d w}\right)_{S p o n}^{L_{w}}\right]$, and this input noise is coupled by the coupling $C_{n}$, and then amplified by a factor $\frac{1}{9} e^{\frac{L_{w}}{L_{G_{n}}}}$ to give the output power spectrum.

The two functions $C_{n}$ and $\lambda_{n}$ are calculated and given in detail by [1], and to a good approximation when $\tilde{a} \geq 0.25$, the calculated result are fit with:

$$
\begin{gathered}
\operatorname{Im}\left(\lambda_{n}\right) \cong \frac{\sqrt{3}}{2} e^{-\frac{1}{\tilde{a} \sqrt{1+\tilde{a}^{2}}}\left(\alpha_{0}+\alpha_{1} \frac{1}{\tilde{a}^{2}}\right)}, \text { and } \\
C_{n}(\tilde{a}) \cong \frac{\sqrt{3}}{\pi \widetilde{a}^{2}} e^{-\frac{1}{\bar{a} \sqrt{1+\bar{a}^{2}}}\left(\beta_{0}+\beta_{1} \frac{1}{\tilde{a}^{2}}\right)}
\end{gathered}
$$

where for the mode $\{1,0\}$, we have $\alpha_{0}=0.397, \alpha_{1}=-0.0067, \beta_{0}=1.093, \beta_{1}=-0.02 ;$ while for the mode $\{1, \pm 1\}$ we have $\alpha_{0}=1.2625, \alpha_{1}=-0.1494, \beta_{0}=5.082, \beta_{1}=-0.5707$.

As an example, let us take $I_{0}=110$ ampere. Using Eq.(5), we find that the Pierce parameter $\rho=8 \times 10^{-3}$, and the scaled beam size $\tilde{a}=0.95$. Applying these to Eq. (6), and 
Eq.(7), we find the power gain lengths and coupling coefficients for the mode $n=\{1,0\}$ to be $L_{G}=0.26 \mathrm{~m}, C=0.24$, and for mode $m=\{1, \pm 1\}, L_{G}=0.43 \mathrm{~m}, C=0.021$.

Using Eq.(4), we can calculate the power spectrum in each mode, and sum over modes. However, empirically, we find it is convenient to calculate the increment of SASE power over spontaneous radiation power ratio by summing over the corresponding ratio increments for all the modes. Thus we have

$$
\begin{aligned}
\frac{\left(\frac{d P}{d \omega}\right)_{S A S E}}{\left(\frac{d P}{d \omega}\right)_{S p m .}^{L_{w}}}-1 & =\left[\frac{1}{9} e^{\frac{1.98}{0.26}}-1\right] \times 0.24 \times \frac{2 \times 0.26}{1.98} \\
& +2 \times\left[\frac{1}{9} e^{\frac{1.98}{0.43}}-1\right] \times 0.021 \times \frac{2 \times 0.43}{1.98}+\ldots \\
& =13.6+0.2+. . \approx 14 .
\end{aligned}
$$

The 1 in each term in the square parentheses is the subtraction of the contribution from the spontaneous radiation. The extra factor 2 in the second term is due to the two modes with $\mathrm{m}= \pm 1$. For higher modes, the gain factor $\frac{1}{9} e^{\frac{L_{w}}{L_{G_{n}}}}$ is rapidly reduced to nearly one or even smaller than one, and the formula eq.(4) is not valid. However, the gain for these higher modes are negligible, this means that they only contribute to the spontaneous radiation, so $\left(\frac{1}{9} e^{\frac{L_{n n}}{L_{G_{n}}}}-1\right)$ for these modes can simply be replaced by 0 as an approximation. The SASE over spontaneous radiation ratio is then $14+1=15$. The SASE power spectrum calculated this way is plotted against the simulation results in Figure 2, showing a good agreement.

Naturally, one familiar with one dimensional high gain FEL theory would raise a question. When the gain is not very high there are three longitudinal terms, i.e., in addition to the growth term, there are other two terms comparable with the growth term: one is exponentially decaying, the other is oscillating; and the formula Eq.(4) is not valid. Why we can still use this formula even the total SASE over spontaneous radiation ratio is rather small, as shown in figure 3 ? 
The answer is that, for the two modes we wrote down in Eq.(8), the gain factor is indeed much larger than one. In three dimensional theory, the corresponding decaying mode and and oscillating mode should be calculated as other transverse modes. They are neglected because we are only calculating gain, and these modes only contribute to the spontaneous radiation, which is subtracted from SASE calculation. If we do not subtract the spontaneous radiation from the SASE, the series would converge very slowly. In addition, for the higher modes, the growth term is not large enough to dominate over the other decaying and oscillating terms, so the calculation becomes very difficult. In short, using guided modes to calculate spontaneous radiation is very difficult and unnecessary. By subtracting the spontaneous radiation, and only calculating the gain, we avoid this difficulty, and obtain an empirically excellent approximation.

Up to now, we used an idealized step function beam profile to test the calculation. To compare with experiment, we use a more realistic waterbag model. We choose the same rms beam size $\sigma_{x}=170 \mu m$. This corresponds a normalized emittance $\varepsilon_{n}=4 m m-m r a d$ for our case with a focusing betatron wavelength $\lambda_{\beta}=3.7 \mathrm{~m}$. We take the local energy spread to be $\left(\frac{\Delta \gamma}{\gamma}\right)_{r m s}=4 \times 10^{-4}$. These parameters lead to a power gain length with waterbag model $L_{G}=0.26 m$, same as the step function model case before.

The calculated results are shown in Figure 3. The dots are the simulation with the waterbad model, and the solid line is the calculation using the analytical method Eq.(8) for the step function model with the same rms beam size. The agreement is very good considering the crude nature of the approximation.

A recent SASE experimental result is plotted on Figure 4. We did not have enough time to characterize the e-beam before this conference. However, the plot clearly shows that the beam quality is better than our originally designed parameters used for our calculation in this paper (110 amp, 4mm-mrad). 


\section{References}

1. L. H. Yu, Phys. Rev. E. (To be published)

2. S. Krinsky, L.H. Yu, Phys. Rev. A 35, 8, 3406, (1987)

3. L. H. Yu, S. Krinsky, Nucl. Instr. Meth. ,A285, 119,(1989)

4. R. Bonifacio, C. Pellegrini, and L. M. Narducci, Opt. Commun. 50, 373 (1984)

5. T.M. Tran, J.S. Wurtele, Computer Phys. Comm. 54, 263 (1989) 
Figure 1. Output power spectrum for spontaneous radiation as a function of wavelength Figure 2. Output power vs current for the step function model

Figure 3. Output power vs current for the waterbag model

Figure 4. Radiation energy vs e-beam charge in the Cornell Wiggler A SASE experiment at BNL 


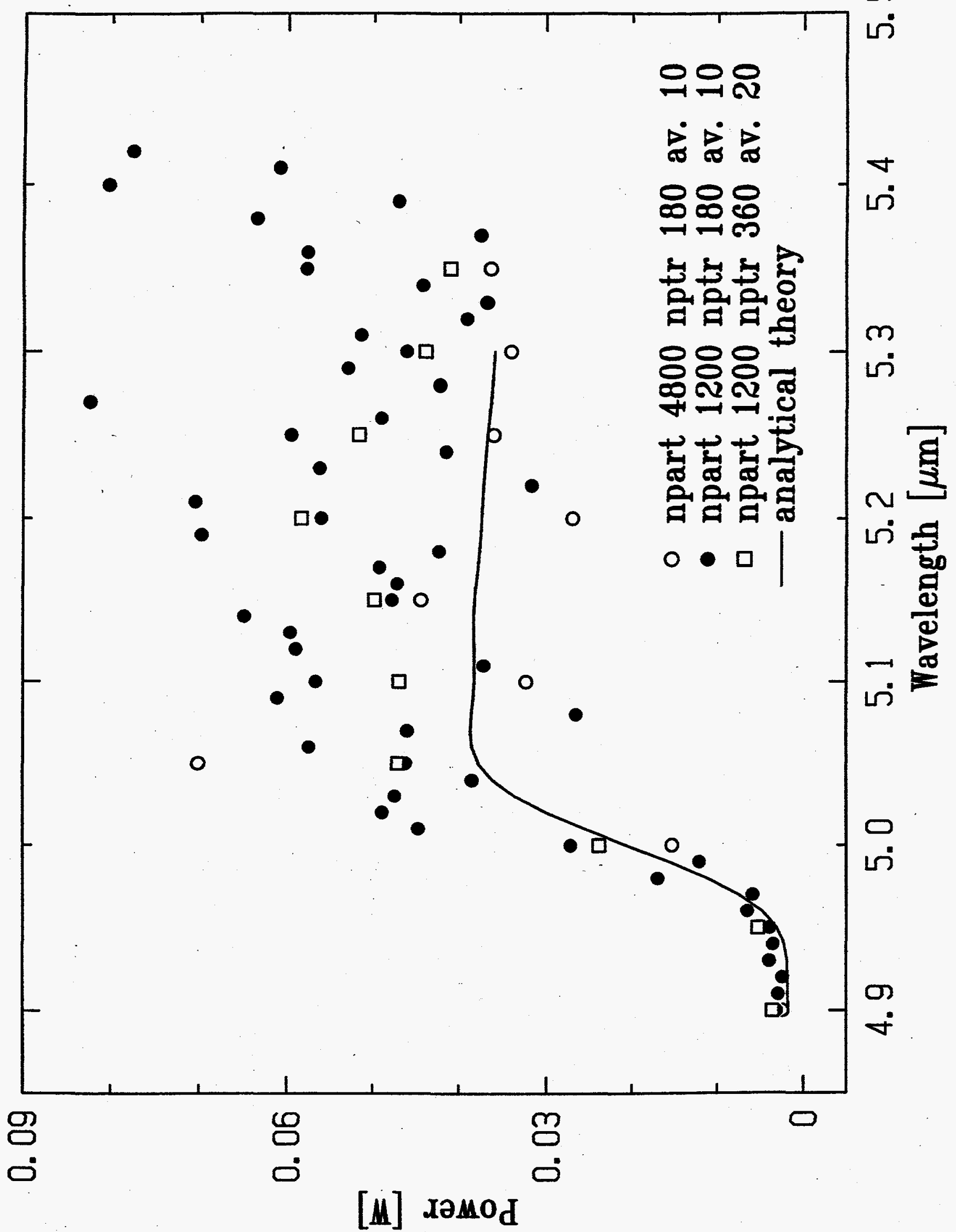




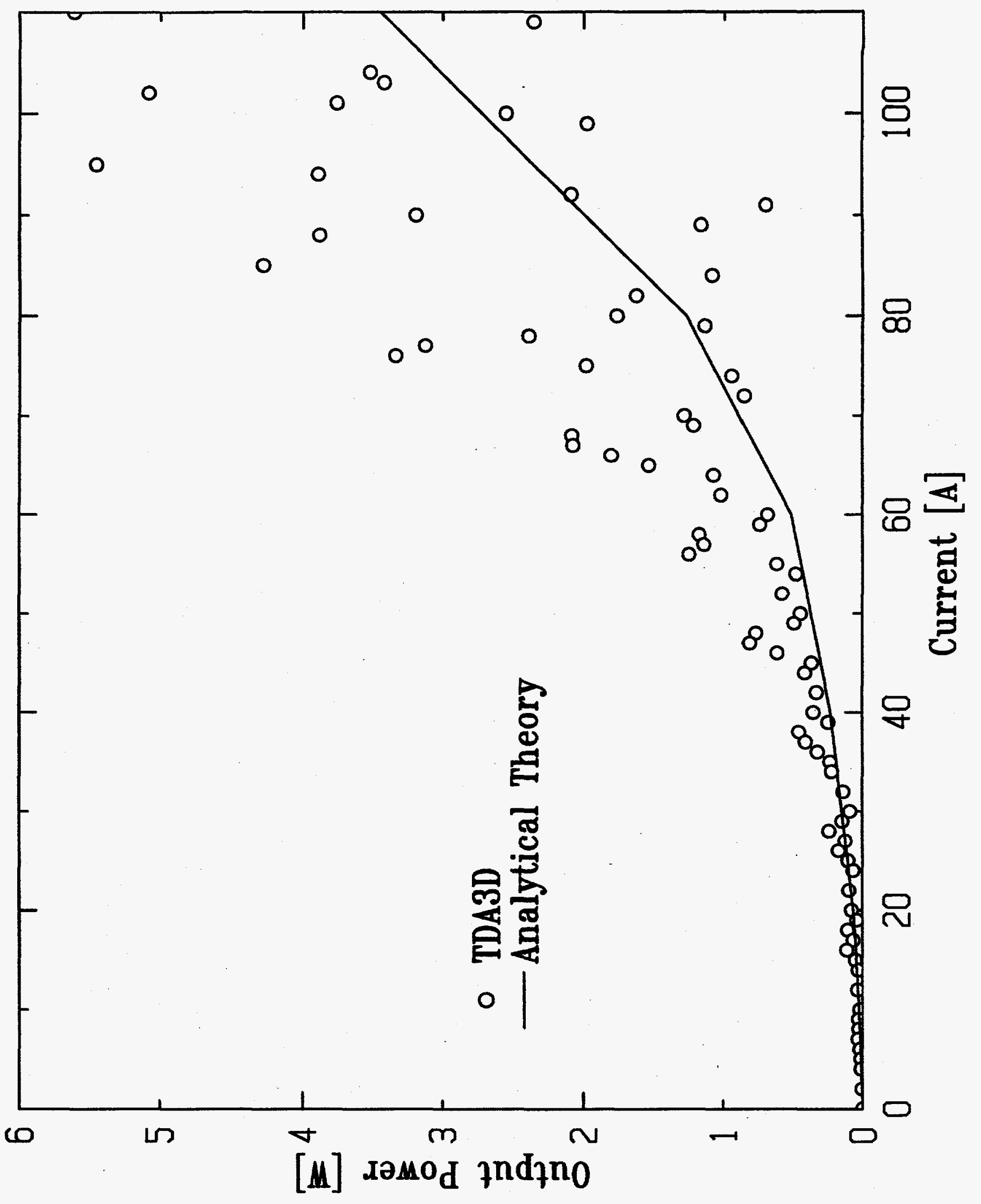

$\stackrel{n}{i}$ 


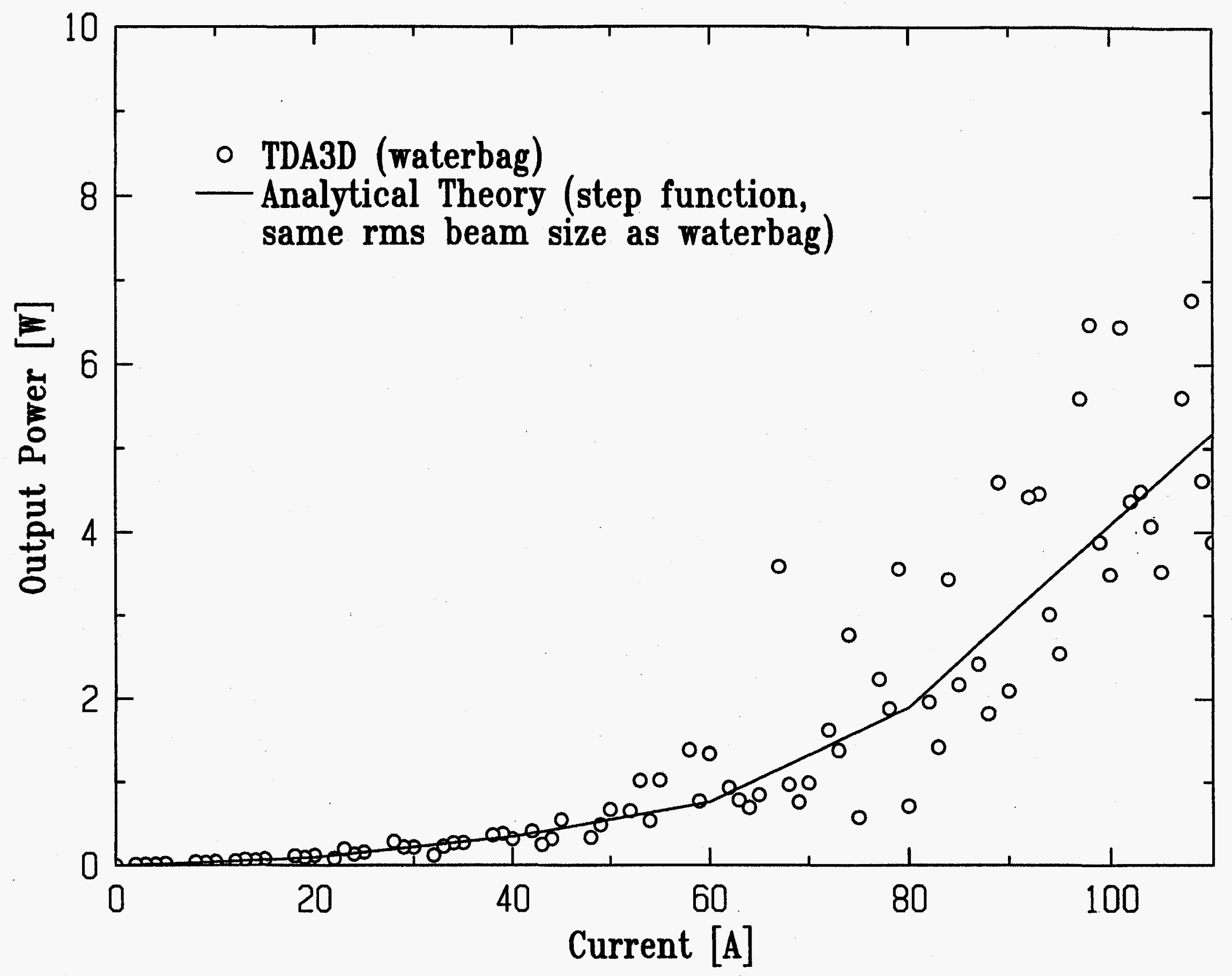




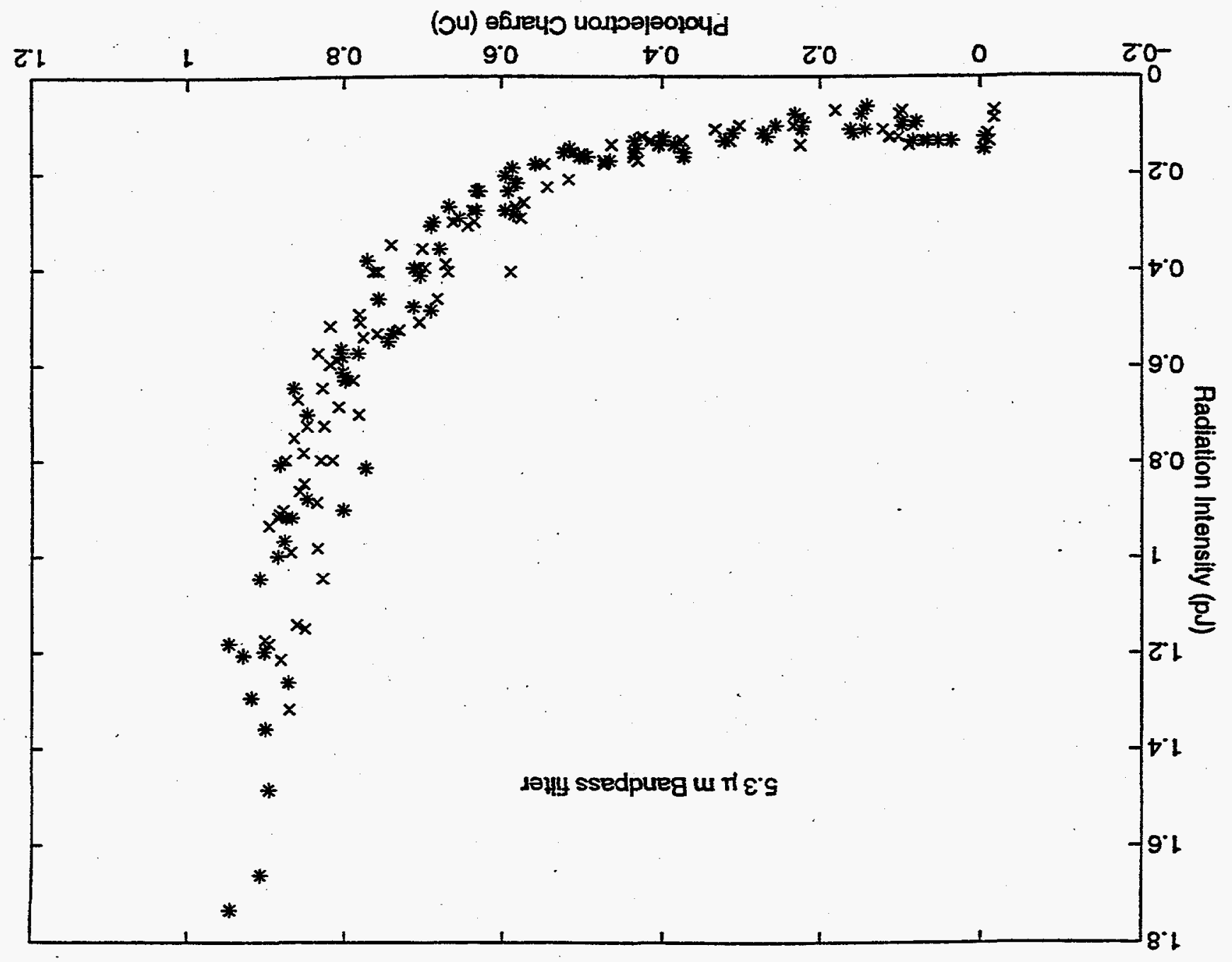

